This is a peer-reviewed, un-copyedited version of an article accepted for publication/published in Nanotechnology 28, 495702. IOP Publishing Ltd is not responsible for any errors or omissions in this version of the manuscript or any version derived from it. The Version of Record is available online at https://doi.org/10.1088/1361-6528/aa926f

This Accepted Manuscript is available for reuse under a CC BY-NC-ND 3.0 licence after the 12 month embargo period provided that all the terms of the licence are adhered to.

(https://creativecommons.org/licenses/by-nc-nd/3.0/) 


\title{
Contraction, cation oxidation state and size effects in cerium oxide nanoparticles
}

Jacopo Stefano Pelli Cresi ${ }^{1,2}$, Maria Chiara Spadaro ${ }^{1,2,3}$, Sergio D’Addato ${ }^{1,2}$, Sergio Valeri ${ }^{1,2}$, Lucia Amidani $^{4}$, Federico Boscherini ${ }^{5,6}$, Giovanni Bertoni ${ }^{7}$, Davide Deiana ${ }^{3}$, Paola Luches ${ }^{2 *}$

${ }^{1}$ Dipartimento di Scienze Fisiche Informatiche e Matematiche, Università degli Studi di Modena e Reggio Emilia, Via G. Campi 213/a, 41125 Modena, Italy

${ }^{2}$ Istituto Nanoscienze, Consiglio Nazionale delle Ricerche, Via G. Campi 213/a, 41125 Modena, Italy

${ }^{3}$ Interdisciplinary Centre for Electron Microscopy, Ecole Polytechnique Fédérale de Lausanne, Station 12, 1015 Lausanne, Switzerland

${ }^{4}$ European Synchrotron Radiation Facility, BP 220, 38043 Grenoble, France

${ }^{5}$ Department of Physics and Astronomy, University of Bologna, Viale C. Berti Pichat 6/2, 40127 Bologna, Italy

${ }^{6}$ Istituto Officina dei Materiali, Consiglio Nazionale delle Ricerche, Operative Group in Grenoble, c/o ESRF, BP 220, 38043 Grenoble, France

${ }^{7}$ Istituto dei Materiali per Elettronica e Magnetismo, Consiglio Nazionale delle Ricerche, Parco Area delle Scienze 37/A, 43124 Parma, Italy

*corresponding author: email: paola.luches@unimore.it

\begin{abstract}
An accurate description of the structural and chemical modifications of cerium oxide nanoparticles is mandatory for understanding their functionality in the applications. In this work we investigate the relation between local atomic structure, oxidation state, defectivity and size in cerium oxide nanoparticles with variable diameter below $10 \mathrm{~nm}$, using X-ray absorption fine structure analysis in the near and extended energy range. The nanoparticles are prepared by physical methods under controlled conditions and analyzed in morphology and crystalline quality by high resolution transmission electron microscopy. We resolve here an important question on the local structure of cerium oxide nanoparticles: we demonstrate a progressive contraction in the $\mathrm{Ce}-\mathrm{O}$ interatomic distance with decreasing nanoparticle diameter and we relate the observed effect to the reduced dimensionality. The contraction is not significantly modified by inducing a $4-6 \%$ higher $\mathrm{Ce}^{3+}$ concentration through thermal annealing in high vacuum. The consequences of the observed average cation-anion distance contraction on the properties of the nanoparticles are discussed.
\end{abstract}

\section{Introduction}

Cerium oxide based materials are attractive due to their capacity to quickly store, release and transport oxygen ions, which makes them suitable for application in different fields. Catalysts based on nanostructured cerium oxide are used for example for the abatement of toxic exhausts from car engines, and they have been shown to be active in several reactions like the water-gas shift, $\mathrm{CO}$ preferential oxidation or methane oxidation reaction [1]. Recent perspective applications include also energy conversion and storage devices, in which nanostructured cerium oxide is proposed as an 
electrode material, for example in fuel cells [2, 3] and supercapacitors [4]. Moreover, cerium oxide nanoparticles have been shown to have a great potential as antioxidant materials for therapeutic purposes [5].

The oxygen storage capacity in cerium oxide is related to the possibility for $\mathrm{Ce}$ ions to reversibly change their oxidation state from $4+$ to $3+$ under mild modifications of the ambient conditions [6]. An atomic scale understanding of the material reducibility and of the possible modifications induced by parameters like size, specific exposed surfaces, and interaction with other materials and molecules is of paramount importance in view of the possibility of designing materials with optimized functionality.

Several studies were dedicated to the investigation of the properties of cerium oxide nanoparticles (NPs) of variable size and shape obtained by different synthesis methods [6-11]. An accurate description of the electronic and atomic structure of cerium oxide as a function of NP size is indeed relevant to properly understand the size-dependent behavior of the material, which was often proved to be unexpectedly different from the bulk. The demonstrated strong dependence of the reducibility and of the oxygen storage capacity on the exposed facet derives strictly from the properties of the different surfaces [12,13]. On the contrary, other properties arising from confinement to the nanometer range have a less trivial explanation; among these we mention as examples the predicted size-dependent oxygen vacancy formation energy, which reaches a minimum at a few nm size [14], and the peculiar delocalization of valence electrons demonstrated for sizes of approximately $2 \mathrm{~nm}$ [15].

An "anomalous" lattice expansion is typically reported when the size of the investigated cerium oxide NPs is below a few tens of nm [8-10, 16-18], as opposed to the contraction reported for metal NPs since the late sixties [19-21]. As the NP diameter decreases, given the higher surface to volume ratio, the concentration of $\mathrm{Ce}^{3+}$ sites, possibly induced on the surface by the chemical synthesis procedures, increases. For this reason the observed lattice expansion has often been suggested to originate from the larger radius of $\mathrm{Ce}^{3+}$ compared to $\mathrm{Ce}^{4+}$ ions $[8,16-18,22,23]$. On the contrary, theoretical studies predict that a lattice contraction is also expected in cerium oxide NPs [23, 24], and ascribe the experimentally observed expansion to the presence of oxygen vacancies [24]. However, a simple correlation between the degree of reduction and the lattice expansion with decreasing cerium oxide NP size has often been questioned, pointing out the modifications which may be induced by the synthesis or measurement conditions $[25,26]$. It is clear that the outstanding question is whether $\mathrm{CeO}_{2}$ NPs contract or expand with decreasing size, demanding for a careful study on NPs with controlled degree of reduction.

Physical methods based on gas phase synthesis have been shown to be suitable for the synthesis of $\mathrm{NP}$ assemblies with a good control over stoichiometry, size and architecture [27-29]. For cerium oxide NPs the oxidation state of part of the Ce ions can be reversibly modified by thermal annealing in vacuum or oxygen pressure $[11,30]$, in close analogy with cerium oxide epitaxial films and nanostructures .

X-ray absorption fine structure (XAFS) allows to simultaneously obtain information on the chemical state of an atomic species and on its local structural environment by analyzing an absorption edge in the near and extended energy range, respectively. In this work we apply the XAFS technique to the $\mathrm{Ce} \mathrm{L}_{3}$ edge in cerium oxide NPs with selected size and controlled degree of reduction, we demonstrate a contraction in the $\mathrm{Ce}-\mathrm{O}$ distance compared to the bulk value and we analyze its dependence on the degree of reduction. 


\section{Experimental methods and data analysis}

The NP samples were obtained using a magnetron sputtering and inert gas aggregation nanocluster source (NC200U, Oxford Applied Research), equipped with a quadrupole mass filter (QMF200, Oxford Applied Research), and installed in an ultrahigh vacuum experimental system connected to an X-ray photoelectron spectroscopy (XPS) apparatus (XR50, Specs double anode X-ray source and Phoibos 150, Specs hemispherical electron analyser) [29, 31]. For the present experiment we used a $99.9 \%$ pure metallic cerium target and a mixture of He and Ar gases in the aggregation region. We selected NPs of variable size, below $10 \mathrm{~nm}$ diameter, by varying the electric power applied to the magnetron target, the Ar and He gas flux in the aggregation region, and the length of the aggregation region. The parameters used for obtaining NPs of different size are summarized in the supporting information, Table S1. The QMF was used to further select the size of the NPs before deposition. The NP deposition rate was evaluated using a quartz microbalance. In the following, the nominal film thickness is defined as the thickness of a continuous cerium oxide film with the same volume as the NP film. The NP films were deposited on a $\mathrm{Si} / \mathrm{SiO}_{\mathrm{x}}$ substrate for XPS and XAS measurements, while carbon coated copper grids were used as a support for transmission electron microscopy (TEM). The samples were deposited in a partial pressure of oxygen $\mathrm{P}_{\mathrm{O}_{2}}=5 \times 10^{-6} \mathrm{mbar}$. In order to maximize the surface oxidation, the samples were exposed to an oxygen partial pressure $\mathrm{P}_{\mathrm{O}_{2}}=6 \times 10^{-5}$ mbar for 30 minutes after the growth. The nominal film thickness was fixed to $10 \mathrm{~nm}$ for the samples to be measured by XAS, while the samples for TEM had a much smaller nominal thickness, in the subnanometer range, in order to be able to identify and analyse individual particles. The TEM measurements were limited to the 4 and $6 \mathrm{~nm}$ NP samples, while only 4, 5 and $9 \mathrm{~nm}$ NP samples were measured by XAS.

All samples were characterized by in-situ XPS, using $\mathrm{Al} \mathrm{K}_{\alpha}$ photons. To obtain quantitative information on the oxidation state the measured Ce $3 \mathrm{~d}$ XPS spectra were fitted using $\mathrm{Ce}^{4+}$, and $\mathrm{Ce}^{3+}$ components, following the procedure introduced by Skála et al [32], already used by our group for the analysis of epitaxial ceria nanostructures $[33,34]$.

Ex-situ scanning TEM images (STEM) in annular dark field mode (ADF) were performed to assess the size distribution and crystalline quality of the NPs. The experiments on the $6 \mathrm{~nm}$ sample were performed using a JEOL JEM-2200FS microscope (IMEM-CNR, Parma), equipped with a Schottky emitter working at $200 \mathrm{keV}$ (point resolution $0.19 \mathrm{~nm}$ ). This instrument was used also to acquire the selected area electron diffraction (SAED) patterns. The experiments on the $4 \mathrm{~nm}$ sample were performed using a double spherical corrected FEI Titan Themis cubed microscope (CIME, EPFL Lausanne) working at $200 \mathrm{keV}$, equipped with an ultra-bright Schottky (XFEG) electron source (point resolution $0.07 \mathrm{~nm}$ ). All the ADF-STEM images have been analysed using the STEM_CELL software [35].

The XAFS measurements were performed at BM08 (LISA) beamline of the European Synchrotron Radiation Facility (ESRF) in Grenoble, France. The measurements were acquired at the $\mathrm{Ce}_{3}$-edge, using a dynamically sagittal-focusing $\mathrm{Si}$ (311) monochromator [36]. In order to eliminate higher-order harmonics, a pair of Pt coated flat mirrors were used to set a cut-off energy of $12 \mathrm{keV}$. The XAFS spectra were acquired in the fluorescence yield mode, using a 12-element hyper-pure Ge detector. The samples were kept in nitrogen atmosphere during the transfer to the synchrotron radiation facility. A sample environment cell was used to perform the reducing thermal annealing, which consists in heating the samples to $1020 \mathrm{~K}$ in high vacuum $\left(\mathrm{P} \sim 10^{-7} \mathrm{mbar}\right)$ for $30 \mathrm{~min}$ using a constant heating 
and cooling rate of $7 \mathrm{~K} / \mathrm{min}$. A similar treatment was previously shown to induce a non-negligible reduction on the surface of cerium oxide ultrathin films and NP [11, 33, 37, 38]. In particular, for this work we used the same cell and the same conditions used in reference [37]. The samples were measured at room temperature (RT) before and after the high-vacuum thermal annealing (indicated as HVT). Two reference samples in powdered form were also measured in transmission mode: $\mathrm{a} \mathrm{CeO}_{2}$ sample, as a reference for the $\mathrm{Ce}^{4+}$ oxidation state, and a $\mathrm{Ce}\left(\mathrm{NO}_{3}\right)_{3} \cdot 6 \mathrm{H}_{2} \mathrm{O}$ sample, as a reference for the $\mathrm{Ce}^{3+}$ oxidation state.

X-ray absorption near edge spectroscopy (XANES) measurements were analysed to have information on the variation of the oxidation state in the samples. In particular, the spectra were fit using five Pearson VII distribution curves, i.e. Lorentz functions raised to a power $\mathrm{m}$, to simulate the near edge features - three related to $\mathrm{Ce}^{4+}$, one related to $\mathrm{Ce}^{3+}$, and one related to the pre-edge - and an arctangent function to reproduce the edge jump, following reference [39]. The energy position of the centre, the FWHM and the shape of the Pearson distributions were fixed to the ones obtained from the fit of the $\mathrm{Ce}^{4+}$ and $\mathrm{Ce}^{3+}$ reference samples (see Figure S1, Supporting Information). Moreover, since the peak originating from $\mathrm{Ce}^{3+}$ ions occurs at a photon energy which is comparable to one of the structures corresponding to $\mathrm{Ce}^{4+}$ ions, the intensity ratio between the first two $\mathrm{Ce}^{4+}$ related features was fixed to the value obtained in the fit of the reference sample (see Figure S1, Supporting Information). Since it is not possible to resolve the $\mathrm{Ce}^{3+}$ and $\mathrm{Ce}^{4+}$ contributions to the pre-edge peak [40], its intensity was not considered in the evaluation of the $\mathrm{Ce}^{3+}$ concentration within the samples. We note that the reference samples for the two Ce oxidation states may contain also ions in the other oxidation state as minority components, located on some of the low coordination sites or at defect sites. This affects the accuracy of the $\mathrm{Ce}^{3+}$ concentration obtained from the fitting. However, all of the considerations made in this work are based on relative variations of the estimated $\mathrm{Ce}^{3+}$ concentration, and they are not affected by the accuracy of the numbers obtained.

The extended X-ray absorption fine structure (EXAFS) was acquired up to $k=9 \AA^{-1}$. The available $k$-range was limited by a small $\mathrm{Cr} \mathrm{K}$ adsorption edge signal, possibly originating from diffused light scattering from the walls of the sample environment cell. The EXAFS spectra were processed using the Athena software package [41]. Starting from the fluorite structure of cerium oxide, the theoretical scattering functions were simulated ab-initio using the FEFF program [42]. Quantitative data analysis was performed by fitting the EXAFS spectra with the simulated scattering functions, including only the Ce-O first shell single scattering path, by means of the Artemis code [43]. The presence of multiple excitations and the mixed valence nature of the excited state [44, 45] has been neglected, given the expected weak intensity of the resulting features and the limited energy range available for the analysis.

\section{Results and Discussion}

A series of cerium oxide NP samples with different nominal diameter were grown with the procedures described in the experimental section. The surface stoichiometry of the NPs after deposition and exposure to oxygen was evaluated by analyzing Ce $3 \mathrm{~d}$ XPS spectra acquired in-situ (Figure 1). The dominant features in the spectra of the samples originate from three doublets corresponding to emission from $\mathrm{Ce}^{4+}$ ions with different final states, indicated as v-u, v' - u', and v' ' - u' ' in Figure 1, following Romeo et al. [46]. As the NP diameter decreases the spectra show a progressive increase of intensity in the binding energy region between $\mathrm{v}$ and $\mathrm{v}$ ', and between $\mathrm{u}$ and $\mathrm{u}$ ', corresponding to the most intense v' - u' doublet from $\mathrm{Ce}^{3+}$ ions (Figure 1). The $\mathrm{Ce}^{3+}$ surface concentration in each sample was evaluated by fitting the spectra with $\mathrm{Ce}^{4+}$ and $\mathrm{Ce}^{3+}$ related doublets [32], and the results 
are reported in Table 1 . The $\mathrm{Ce}^{3+}$ surface concentration in the $4 \mathrm{~nm}$ NPs is above $20 \%$ and it decreases with increasing NP diameter, in agreement with the presence of a higher concentration of lowcoordinated surface sites in NP with smaller diameter. It has to be noted that the accuracy obtained by this method is limited to approximately $\pm 10 \%$. Moreover, the measured values may well be systematically overestimated due to a demonstrated photon beam-induced surface reduction of the NP surface in ultra-high vacuum [11].

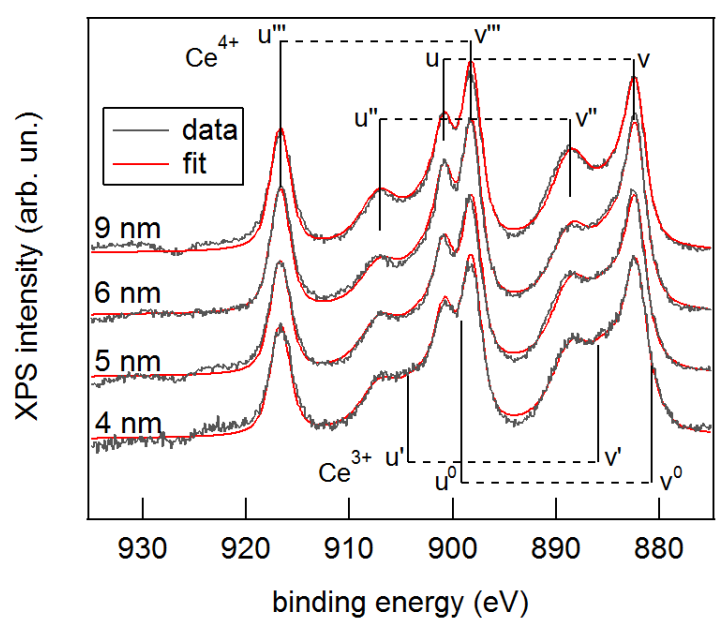

Figure 1: Ce 3d XPS spectra (black curve) of cerium oxide NPs with different average diameter. The fitting using Ce $\mathrm{e}^{3+}$ and $\mathrm{Ce}^{4+}$ related components are also shown (red curve). The binding energy positions of the $\mathrm{Ce}^{4+}-$ and of the $\mathrm{Ce}^{3+}-$ related doublets are indicated in the top and bottom of the figure, respectively.

\begin{tabular}{|l|l|}
\hline$\varnothing(\mathbf{n m})$ & $\mathbf{C e}^{3+}(\%)$ \\
\hline 4 & $23 \pm 2$ \\
\hline 5 & $18 \pm 2$ \\
\hline 6 & $14 \pm 2$ \\
\hline 9 & $10 \pm 2$ \\
\hline
\end{tabular}

Table 1: $\mathrm{Ce}^{3+}$ surface concentration in the cerium oxide NP samples of different diameter, evaluated from the fitting of Ce $3 \mathrm{~d}$ XPS spectra. The uncertainties represent the precision of the $\mathrm{Ce}^{3+}$ concentration values, rather than their accuracy.

The morphology, crystal quality and size distribution of the cerium oxide NPs were investigated by high resolution STEM measurements. A representative image for NPs with selected diameter of $6 \mathrm{~nm}$ is shown in Figure 2 a. The corresponding high resolution image, shown in the figure inset, shows that most of the NPs have a single-crystalline fluorite structure ( $\mathrm{Fm} \overline{3} \mathrm{~m}$ space group) with evident (111) crystal planes fringes. 

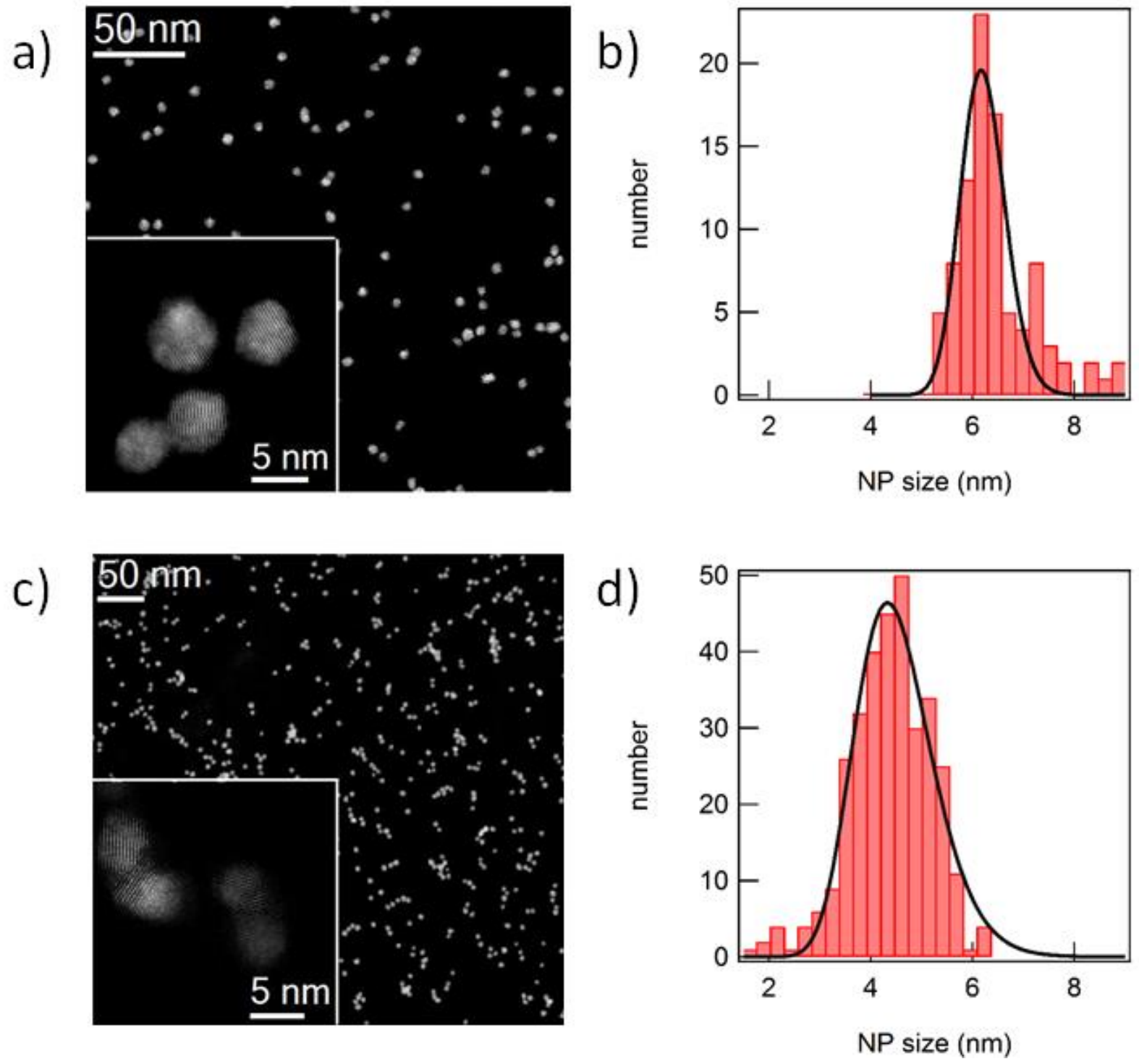

Fig.2: ADF-STEM images of cerium oxide NPs of $6 \mathrm{~nm}$ (a) and $4 \mathrm{~nm}$ (c) average diameter; insets: higher magnification of selected NPs; b) and d) size distribution extracted from the STEM images in a) and c), respectively, and fit with a lognormal distribution.

The shape of the NPs is polyhedral and the (111) and (100) surfaces are preferentially exposed. Most of the NPs are single crystalline, while few of them are polycrystalline, as already observed in previous work on NP grown in the same way [30]. The size distribution of the $6 \mathrm{~nm}$ NP sample (Figure $2 \mathrm{~b}$ ) is rather narrow; a fit with a log-normal distribution has the maximum at $6.2 \mathrm{~nm}$ and a full width at half maximum (FWHM) of $1.1 \mathrm{~nm}$. The STEM images corresponding to NPs with selected diameter of $4 \mathrm{~nm}$, shown in Figure $2 \mathrm{c}$, confirm that even the NPs with smallest size analyzed in this work, maintain their individuality and the high degree of crystal quality. In this case the size distribution (Figure $2 \mathrm{~d}$ ) has the maximum at $4.3 \mathrm{~nm}$ and it is slightly broader (FWHM=1.7 $\mathrm{nm}$ ) than the distribution of the $6 \mathrm{~nm}$ sample. The different width of the size distributions is due to the values of mass resolution adopted for the QMF in the two samples $(U / V=0.100$ for the $6 \mathrm{~nm}$ sample, $U / V$ $=0.069$ for the $4 \mathrm{~nm}$ sample). This choice results from a compromise between an appreciable NP deposition rate and a small dispersion of the NP size. The TEM-SAED patterns are compatible with the fluorite structure (see figure S3). However, we could not extract precise information on the long range lattice parameters from the positions of the rings, which appeared weak and broad, given the high dilution of the nanoparticles and their small size. 
The Ce $\mathrm{L}_{3}$-edge XANES spectra of the cerium oxide NP samples before and after the thermal annealing are shown in Figure 3. For all samples the XANES line shape shows dominant features which originate from $\mathrm{Ce}^{4+}$ ions, namely a peak at $5732.6 \mathrm{eV}$ (peak B in Figure 3) ascribed to a screened transition from $2 \mathrm{p}$ levels to the $5 \mathrm{~d}$ band, and one at $5739.6 \mathrm{eV}$ (peak A in Figure 3 ) ascribed to unscreened transitions between the same levels [47]. In addition, the spectra show a low energy shoulder at $5728.2 \mathrm{eV}$ (peak $\mathrm{C}$ in Figure 3) ascribed to crystal-field splitting of the $5 \mathrm{~d}$ band [48] and a poorly resolved pre-edge feature (peak D in Figure 3 ) ascribed to dipole-forbidden $2 \mathrm{p}-4 \mathrm{f}$ transitions [49].

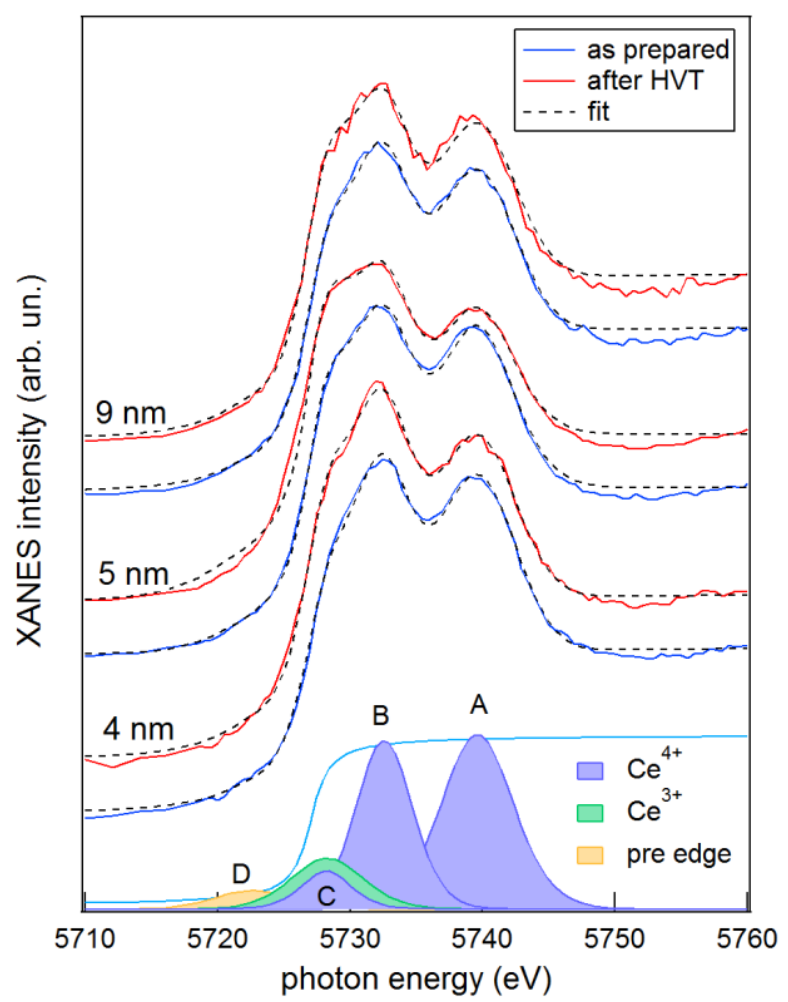

Figure 3: $\mathrm{Ce}_{3}$-edge XANES spectra of the cerium oxide NP samples before (blue curves) and after (red curves) the reducing high vacuum thermal annealing (HVT). The fits of the spectra, using the $\mathrm{Ce}^{4+}$ and $\mathrm{Ce}^{3+}$ related components shown at the bottom, are also reported (dashed line).

After the thermal annealing at $1020 \mathrm{~K}$ in vacuum the edge is slightly shifted towards lower photon energies and the shape of the first feature changes, in agreement with a non-negligible degree of reduction of the samples. The $\mathrm{Ce}^{4+}$ and $\mathrm{Ce}^{3+}$ concentrations in the samples were quantified by fitting the spectra with $\mathrm{Ce}^{4+}$ and $\mathrm{Ce}^{3+}$ components and the results are shown in Table 2. We note that also in this case all the considerations made are based on the variation of the degree of reduction in the different samples, rather than on their absolute values, which can be affected by a non-negligible inaccuracy, and possibly systematically overestimated due to eventual beam-induced surface reduction.

\begin{tabular}{|l|l|l|}
\hline$\varnothing(\mathrm{nm})$ & $\begin{array}{l}\mathrm{Ce}^{3+}(\%) \\
\text { as prepared }\end{array}$ & $\begin{array}{l}\mathrm{Ce}^{3+}(\%) \\
\text { after HVT }\end{array}$ \\
\hline 4 & $9 \pm 1$ & $13 \pm 1$ \\
\hline 5 & $13 \pm 1$ & $19 \pm 1$ \\
\hline
\end{tabular}




\begin{tabular}{|l|l|l|}
\hline 9 & $13 \pm 1$ & $17 \pm 1$ \\
\hline
\end{tabular}

Table 2: $\mathrm{Ce}^{3+}$ concentration in the cerium oxide NP samples of different diameter, evaluated from the fitting of Ce $\mathrm{L}_{3}$ edge XANES spectra, before and after the reducing high vacuum thermal treatment (HVT) at $1020 \mathrm{~K}$. The uncertainties represent the precision of the $\mathrm{Ce}^{3+}$ concentration values, rather than their accuracy.

In spite of this, the relatively low degree of reduction detected by XANES measurements on the cerium oxide NPs before the reducing thermal treatment indicates that the bulk of the NPs is well oxidized, although the $\mathrm{Ce}^{3+}$ species represent a non-negligible minority component. The comparison with the systematically higher values of $\mathrm{Ce}^{3+}$ concentration detected by XPS, which is a surface sensitive technique, indicates that the $\mathrm{Ce}^{3+}$ species are mainly localized at the surface. The slightly higher $\mathrm{Ce}^{3+}$ concentration in the samples with larger diameter possibly reflects a slightly higher bulk defectivity in the larger samples. The reducing thermal annealing induces a moderate increase of the $\mathrm{Ce}^{3+}$ concentration in all samples. The difference between the $\mathrm{Ce}^{3+}$ concentration before and after the treatment is comparable in the three samples within the error, which indicates that the process induces a comparable fraction of $\mathrm{Ce}^{3+}$ ions independent on their size and on the initial defectivity. A similar thermal annealing treatment in ultra-high vacuum has been previously shown to induce a relevant reduction of the NP surface $[11,30]$. The mild reduction observed by XANES, which is sensitive to the whole NP volume, is an indication that the reduction process involves mainly the NP surface.

To have information on the local atomic structure of the NPs with controlled oxidation state, the EXAFS energy region of the spectra was acquired right after the XANES for each sample. The magnitude of the Fourier transforms of the EXAFS spectra of the NP samples before and after the high-vacuum thermal annealing are shown in Figure 4. 


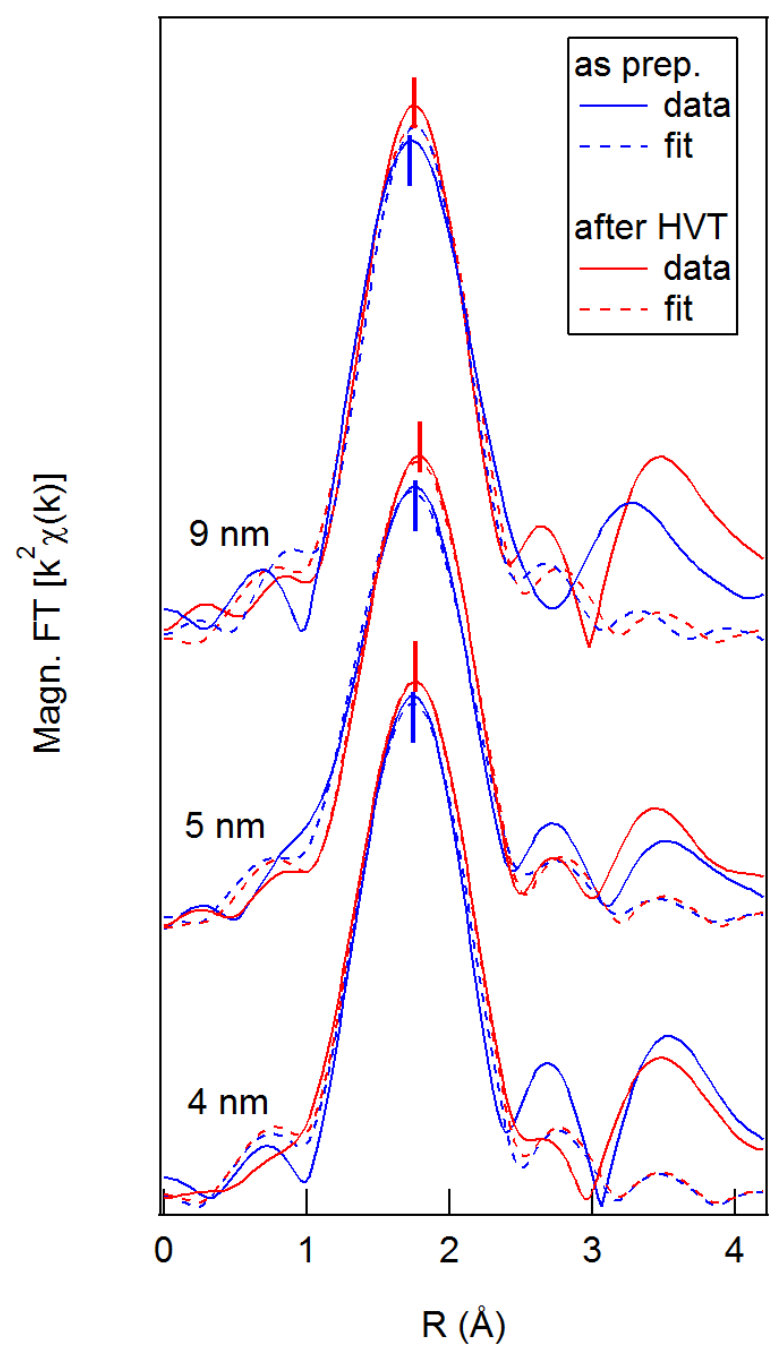

Figure 4: Magnitude of Fourier transforms of $\mathrm{Ce}_{3}$-edge EXAFS spectra before (blue curves) and after (red curves) the reducing high vacuum thermal treatment (HVT) for three different cerium oxide NP samples. The position of the peak maximum is marked with blue and red ticks. The fits are also reported as dashed lines.

A peak around $1.8 \AA$, marked with a blue tick in Figure 4, corresponding to single scattering from first shell oxygen atoms, is the dominant feature in the spectra of the as prepared samples. After the thermal annealing the peak, marked with a red tick in Figure 4, is systematically shifted towards larger $\mathrm{Ce}-\mathrm{O}$ distances and its amplitude is slightly increased. A first-shell fitting allowed to have quantitative information on the local structure of the different samples and on the evolution with the high-vacuum thermal annealing. In the fitting the many body amplitude reduction factor was fixed to the value obtained from the fit of the reference $\mathrm{CeO}_{2}$ sample spectrum (see Figure $\mathrm{S} 2$, supporting information). The free fitting parameters were the energy origin shift, the coordination number, the variation of the Ce-O interatomic distance, and the Debye-Waller factor. The fitting was performed in the ranges $k=$ 2.5 - $7.0 \AA^{-1}$ and $R=1-3 \AA$ on $\mathrm{k}^{2}$-weighted spectra. The results obtained are summarized in Table 3, which reports also the values obtained on the reference $\mathrm{CeO}_{2}$ sample, using the same $k$ and $R$ range.

\begin{tabular}{|l|l|l|l|l|l|}
\hline$\varnothing(\mathrm{nm})$ & treatment & $\mathbf{N}$ & $\boldsymbol{\sigma}\left(\mathbf{\AA}^{\mathbf{2}}\right)$ & $\mathbf{E} \mathbf{( e V )}$ & $\boldsymbol{\Delta} \mathbf{R}(\mathbf{C e}-\mathbf{O})(\AA)$ \\
\hline 9 & as prepared & $7(2)$ & $0.007(7)$ & $3(2)$ & $-0.06(3)$ \\
\hline 9 & after HVT & $7.5(1.5)$ & $0.006(4)$ & $2.4(1.7)$ & $-0.08(2)$ \\
\hline 5 & as prepared & $7.6(1.2)$ & $0.011(4)$ & $2.1(1.3)$ & $-0.084(19)$ \\
\hline 5 & after HVT & $6.1(5)$ & $0.005(2)$ & $3.0(7)$ & $-0.061(9)$ \\
\hline 4 & as prepared & $7(2)$ & $0.006(6)$ & $2(2)$ & $-0.08(3)$ \\
\hline
\end{tabular}




\begin{tabular}{|l|l|l|l|l|l|}
\hline 4 & after HVT & $7.8(9)$ & $0.008(3)$ & $2.8(1.0)$ & $-0.076(14)$ \\
\hline $\mathrm{CeO}_{2}$ reference & -- & 8 & $0.006(3)$ & $4.7(1.1)$ & $-0.046(16)$ \\
\hline
\end{tabular}

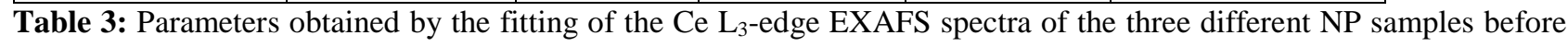
and after the high-vacuum thermal annealing (HVT): coordination numbers $(\mathrm{N})$, Debye-Waller factors $(\sigma)$, energy origin shift $\left(\mathrm{E}_{0}\right)$, and variation of the $\mathrm{Ce}-\mathrm{O}$ interatomic distance $(\Delta \mathrm{R}(\mathrm{Ce}-\mathrm{O}))$. The values obtained for the $\mathrm{CeO}_{2}$ reference sample are also reported. The numbers in parentheses indicate the standard deviation on the last or last two digits.

The correlation between the coordination numbers and the Debye-Waller factors in the fitting procedure prevents from drawing reliable conclusions on the evolution of the two parameters with the NP diameter and high-vacuum thermal annealing. We note that the obtained values are not very different from the ones in the reference $\mathrm{CeO}_{2}$ sample, within the error bars, confirming the dominant fluorite bulk-like coordination of Ce ions in the NPs. On the contrary, the observed values for the Ce$\mathrm{O}$ distance deserve some considerations. The NP samples show a small, but systematic, compression of the Ce-O interatomic distance with decreasing NP size. The measured contraction as a function of the reciprocal average diameter of the NPs is reported in Figure 5.

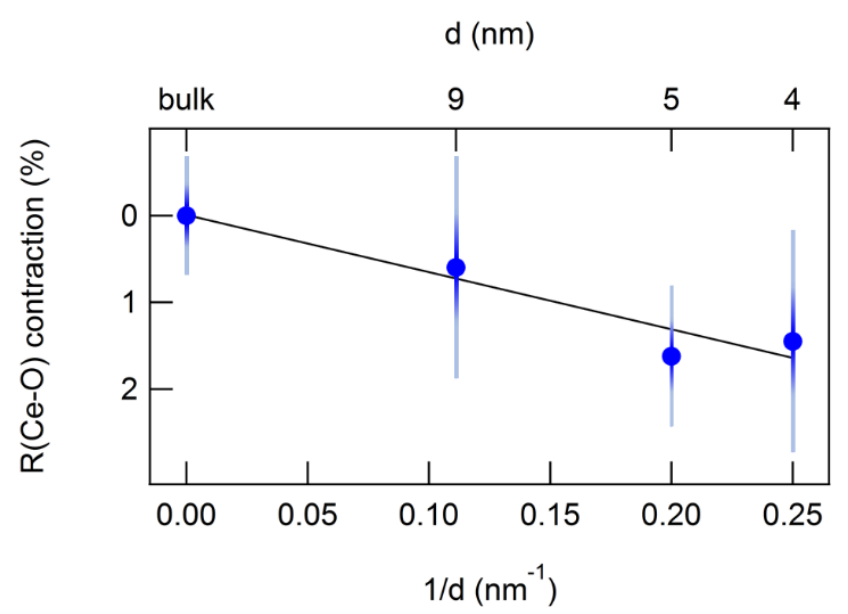

Figure 5: Contraction of the Ce-O interatomic distance evaluated from the fitting of the $\mathrm{Ce}_{3}$ edge EXAFS spectra of the NPs of different diameter and of the $\mathrm{CeO}_{2}$ reference sample as function of the reciprocal average diameter. The solid line represents a linear fit of the data. The error bars reported in graded color indicate the standard deviations on the Ce$\mathrm{O}$ distance obtained from the fitting.

The majority of previous works on the structure of cerium oxide NPs report a progressive lattice expansion with decreasing particle diameter between 2 and $10 \mathrm{~nm}$. The expansion has been frequently ascribed to a higher concentration of $\mathrm{Ce}^{3+}$ ions at smaller NP size, and to the larger ionic radius of $\mathrm{Ce}^{3+}$ with respect to $\mathrm{Ce}^{4+}$. A recent work by Paun et al. proved that the modifications of lattice parameter are not fully correlated with the NP stoichiometry, since also well oxidized NPs showed a non-negligible lattice expansion, ascribed to the negative Madelung pressure at reduced dimensionality [50]. Assuming the thermodynamic model developed by Vermaak [19], which relates the relative volume variation $\Delta \mathrm{V} / \mathrm{V}$ to the reciprocal radius $1 / \mathrm{r}$ with a linear dependence involving the surface stress $\mathrm{f}$, and the bulk modulus $\mathrm{B}\left(\frac{\Delta V}{V}=-\frac{2 f}{B} \frac{1}{r}\right)$, other works ascribe the observed expansion to a negative surface stress, in analogy with metallic NPs, although in the latter case the surface stress is positive and it leads to a lattice contraction. The strong dependence of the lattice parameter on the synthesis methods and on the measurement conditions has been discussed in detail . In a theoretical study Loschen et al. predicted a contraction of the average interatomic distance in cerium oxide clusters with size of approximately 1-2 nm without oxygen vacancies, and they ascribed the interatomic distance modification to dimensional effects, in close analogy to the behavior observed for metallic NPs . At present, only two works reported an experimental evidence for interatomic contraction in cerium oxide NPs. Nachimuthu et al. observed a contraction in NPs with 
diameter below $15 \mathrm{~nm}$ and they related it to the decrease in coordination of Ce ions [39]. In another work, only NPs with diameter above $15 \mathrm{~nm}$ showed an increasing lattice contraction with decreasing diameter, while smaller NP exhibited a reverse trend, due to a dominant lattice expansion induced by oxygen vacancies . Moreover, an almost isotropic contraction of the $\mathrm{Ce}-\mathrm{O}$ distance was observed on epitaxial cerium oxide nanostructures of a few tens of nm lateral size and $\sim 1 \mathrm{~nm}$ thickness [51]. In this case the in-plane contraction was ascribed to epitaxial effects, while the out-of-plane contraction, unexpected considering the bulk elastic theory, was hypothesized to originate from reduced dimensionality effects. In the present study, we could measure simultaneously the cerium local structural environment and its oxidation state. We detected a non-negligible Ce-O contraction even in NPs with a small concentration of $\mathrm{Ce}^{3+}$ sites. Figure 5 shows that the Ce-O bond length has a linear dependence on the surface to volume ratio $S / V$, in turn proportional to the reciprocal diameter $1 / d$. This is a clear indication that the contraction is a dimensionality related effect, in close analogy with metallic NPs and in agreement with theoretical predictions [23, 24]. Our data demonstrate that in cerium oxide NPs the dimensionality-induced bulk contraction dominates over the expected local expansion around $\mathrm{Ce}^{3+}$ sites, resulting in an overall decrease of the average $\mathrm{Ce}-\mathrm{O}$ distance. We believe that the clean conditions used in the preparation and measurement of the NPs and the low concentration of defects allowed to clearly identify the dimensionality-induced contraction. Note that diffraction techniques, often employed to determine the lattice variation in previous studies of cerium oxide NP, are only sensitive to well-ordered crystalline phases, while the XAFS technique used in the present study detects the average $\mathrm{Ce}-\mathrm{O}$ distance in all the $\mathrm{Ce}$-containing phases present in the sample, including possible less ordered phases. The assumption of a dimensionality-related origin of the observed contraction is strengthened by the fact that defective low-ordered zones, eventually present in the samples, are expected to have an expanded $\mathrm{Ce}-\mathrm{O}$ distance with respect to the bulk value. The high-vacuum thermal annealing, observed to induce a moderate increase of $\mathrm{Ce}^{3+}$ concentration, does not significantly modify the observed contraction within the uncertainties in the measurements (Table 4), confirming that dimensionality is largely dominant over stoichiometry-related effects.

The contraction in cation-anion distance is indeed expected to modify some of the properties of the NPs, since it originates from a stronger average bonding. In general, a different band structure is expected, with consequences e.g. on the optical properties [52], or on the mechanical properties [53]. A possibly lower mobility of oxygen ions and electrons may also be induced. The lower electron mobility may be an advantage for some applications, like for example fuel cell electrodes in which a low electric conductivity may be required. On the other hand, a lower oxygen ion mobility will certainly limit the oxygen storage capacity, which is typically required to be maximized in most applications. In spite of this, we do not foresee a reduced surface reactivity in the NPs here investigated, due to the low coordination of the cerium ions on the surface, and the non-negligible degree of surface reduction measured in vacuum, which is expected to favor the reversible uptake and release of oxygen ions.

\section{Conclusions}

Cerium oxide NPs with diameter below $10 \mathrm{~nm}$ have a dominant single crystalline fluorite structure and they show a contraction of the Ce-O distance compared to the bulk value, in contrast with the frequently observed lattice expansion with decreasing NP size. The contraction has a linear dependence on the surface-to-volume ratio and it is ascribed to a compressive strain arising from reduced dimensionality. The size-induced strain dominates over the local expansion expected at the $\mathrm{Ce}^{3+}$ sites, due to the low concentration of reduced ions in the investigated samples. The results here 
described can help to understand the relationship between structure and functionality in systems based on cerium oxide NPs.

\section{Acknowledgements}

The authors wish to thank the European Synchrotron Radiation Facility for provision of beamtime and the staff of the BM08 beamline, in particular Francesco D'Acapito and Alessandro Puri, for their excellent support during the setup of the XAFS experiment and during the measurements.

The work was performed with the financial support of MIUR through the PRIN project “2015CL3APH - NEWLI".

\section{References}

1. Trovarelli A 1996 Catalytic Properties of Ceria and $\mathrm{CeO}_{2}$-Containing Materials. Catalysis Reviews 38 439-520

2. Mori T, Ou DR, Zou J, Drennan J 2012 Present status and future prospect of design of Pt-cerium oxide electrodes for fuel cell applications. Progress in Natural Science: Materials International 22 561-71 3. Fiala R, Vaclavu M, Rednyk A, Khalakhan I, Vorokhta M, Lavkova J, et al. 2015 Pt-CeOx thin film catalysts for PEMFC. Catal Today 240, Part B 236-41

4. Maiti S, Pramanik A, Mahanty S 2014 Extraordinarily high pseudocapacitance of metal organic framework derived nanostructured cerium oxide. Chemical Communications 50 11717-20

5. Celardo I, Pedersen JZ, Traversa E, Ghibelli L 2011 Pharmacological potential of cerium oxide nanoparticles. Nanoscale 3 1411-20

6. Trovarelli A FPs 2013 Catalysis by Ceria and Related Materials London Imperial College Pr.

7. Mai H-X, Sun L-D, Zhang Y-W, Si R, Feng W, Zhang H-P, et al. 2005 Shape-Selective Synthesis and Oxygen Storage Behavior of Ceria Nanopolyhedra, Nanorods, and Nanocubes. The Journal of Physical Chemistry B 109 24380-5

8. Tsunekawa S, Sivamohan R, Ito S, Kasuya A, Fukuda T 1999 Structural study on monosize $\mathrm{CeO}_{2-x}$ nano-particles. Nanostructured Materials 11 141-7

9. Zhang F, Chan S-W, Spanier JE, Apak E, Jin Q, Robinson RD, et al. 2002 Cerium oxide nanoparticles: Size-selective formation and structure analysis. Appl Phys Lett 80 127-9

10. Zou H, Lin YS, Rane N, He T 2004 Synthesis and Characterization of Nanosized Ceria Powders and High-Concentration Ceria Sols. Industrial \& Engineering Chemistry Research 43 3019-25

11. Spadaro MC, D'Addato S, Gasperi G, Benedetti F, Luches P, Grillo V, et al. 2015 Morphology, structural properties and reducibility of size-selected $\mathrm{CeO}_{2-x}$ nanoparticle films. Beilstein Journal of Nanotechnology 6 60-7

12. Conesa JC 1995 Computer Modeling of Surfaces and Defects on Cerium Dioxide. Surf Sci 339 337-52

13. Sayle TXT, Parker SC, Catlow CRA 1994 The role of oxygen vacancies on ceria surfaces in the oxidation of carbon monoxide. Surf Sci 316 329-36

14. Migani A, Vayssilov GN, Bromley ST, Illas F, Neyman KM 2010 Dramatic reduction of the oxygen vacancy formation energy in ceria particles: a possible key to their remarkable reactivity at the nanoscale. $J$ Mater Chem 20 10535-46

15. Cafun JD, Kvashnina KO, Casals E, Puntes VF, Glatzel P 2013 Absence of $\mathrm{Ce}^{3+}$ Sites in Chemically Active Colloidal Ceria Nanoparticles. Acs Nano 7 10726-32

16. Deshpande S, Patil S, Kuchibhatla SV, Seal S 2005 Size dependency variation in lattice parameter and valency states in nanocrystalline cerium oxide. Appl Phys Lett 87133113

17. Wu L, Wiesmann HJ, Moodenbaugh AR, Klie RF, Zhu Y, Welch DO, et al. 2004 Oxidation state and lattice expansion of $\mathrm{CeO}_{2-x}$ nanoparticles as a function of particle size. Phys Rev B 69125415 
18. Baranchikov AE, Polezhaeva OS, Ivanov VK, Tretyakov YD 2010 Lattice expansion and oxygen nonstoichiometry of nanocrystalline ceria. CrystEngComm 12 3531-3

19. Mays CW, Vermaak JS, Kuhlmann-Wilsdorf D 1968 On surface stress and surface tension: II. Determination of the surface stress of gold. Surf Sci 12 134-40

20. Apai G, Hamilton JF, Stohr J, Thompson A 1979 Extended X-Ray Absorption Fine Structure of Small $\mathrm{Cu}$ and Ni Clusters: Binding-Energy and Bond-Length Changes with Cluster Size. Phys Rev Lett 43 165-9

21. Montano PA, Schulze W, Tesche B, Shenoy GK, Morrison TI 1984 Extended x-ray-absorption finestructure study of Ag particles isolated in solid argon. Phys Rev $B 30$ 672-7

22. Tsunekawa S, Ishikawa K, Li ZQ, Kawazoe Y, Kasuya A 2000 Origin of Anomalous Lattice Expansion in Oxide Nanoparticles. Phys Rev Lett 85 3440-3

23. Cordatos H, Ford D, Gorte RJ 1996 Simulated Annealing Study of the Structure and Reducibility in Ceria Clusters. The Journal of Physical Chemistry 100 18128-32

24. Loschen C, Bromley ST, Neyman KM, Illas F 2007 Understanding ceria nanoparticles from firstprinciples calculations. J Phys Chem C 111 10142-5

25. Paun C, Safonova OV, Szlachetko J, Abdala PM, Nachtegaal M, Sa J, et al. 2012 Polyhedral $\mathrm{CeO}_{2}$ Nanoparticles: Size-Dependent Geometrical and Electronic Structure. The Journal of Physical Chemistry $C$ $1167312-7$

26. Chen L, Fleming P, Morris V, Holmes JD, Morris MA 2010 Size-Related Lattice Parameter Changes and Surface Defects in Ceria Nanocrystals. The Journal of Physical Chemistry C 114 12909-19

27. Wegner K, Piseri P, Tafreshi HV, Milani P 2006 Cluster beam deposition: a tool for nanoscale science and technology. Journal of Physics D: Applied Physics 39 R439

28. Martinez L, Mayoral A, Espineira M, Roman E, Palomares FJ, Huttel Y 2017 Core@shell, Au@TiOx nanoparticles by gas phase synthesis. Nanoscale

29. D'Addato S, Spadaro MC, Luches P, Grillo V, Frabboni S, Valeri S, et al. 2014 Controlled growth of $\mathrm{Ni} / \mathrm{NiO}$ core-shell nanoparticles: Structure, morphology and tuning of magnetic properties. App/ Surf Sci $3062-6$

30. Spadaro MC, Luches P, Bertoni G, Grillo V, Turner S, Van Tendeloo G, et al. 2016 Influence of defect distribution on the reducibility of $\mathrm{CeO}_{2-x}$ nanoparticles. Nanotechnology 27425705

31. D'Addato S, Grillo V, Altieri S, Frabboni S, Rossi F, Valeri S 2011 Assembly and Fine Analysis of $\mathrm{Ni} / \mathrm{MgO}$ Core/Shell Nanoparticles. The Journal of Physical Chemistry C 115 14044-9

32. Skála T, Šutara F, Škoda M, Prince KC, Matolín V 2009 Palladium interaction with $\mathrm{CeO} 2$, Sn-Ce-O and Ga-Ce-O layers. Journal of Physics: Condensed Matter 21055005

33. Luches P, Pagliuca F, Valeri S 2011 Morphology, Stoichiometry, and Interface Structure of $\mathrm{CeO}_{2}$ Ultrathin Films on Pt(111). J Phys Chem C 115 10718-26

34. Luches P, Pagliuca F, Valeri S 2014 Structural and morphological modifications of thermally reduced cerium oxide ultrathin epitaxial films on Pt(111). Phys Chem Chem Phys 16 18848-57

35. Grillo V, Rotunno E 2013 STEM_CELL: A software tool for electron microscopy: Part I-simulations. Ultramicroscopy 125 97-111

36. Pascarelli S, Boscherini F, D'Acapito F, Hrdy J, Meneghini C, Mobilio S 1996 X-ray Optics of a Dynamical Sagittal-Focusing Monochromator on the GILDA Beamline at the ESRF. Journal of Synchrotron Radiation 3 147-55

37. Gasperi G, Amidani L, Benedetti F, Boscherini F, Glatzel P, Valeri S, et al. 2016 Electronic properties of epitaxial cerium oxide films during controlled reduction and oxidation studied by resonant inelastic X-ray scattering. Phys Chem Chem Phys 1820511

38. Eck S, Castellarin-Cudia C, Surnev S, Ramsey MG, Netzer FP 2002 Growth and thermal properties of ultrathin cerium oxide layers on $\mathrm{Rh}(111)$. Surf Sci 520 173-85

39. Nachimuthu P, Shih W-C, Liu R-S, Jang L-Y, Chen J-M 2000 The Study of Nanocrystalline Cerium Oxide by X-Ray Absorption Spectroscopy. Journal of Solid State Chemistry 149 408-13

40. Kvashnina KO, Butorin SM, Glatzel P 2011 Direct study of the f-electron configuration in lanthanide systems. Journal of Analytical Atomic Spectrometry 26 1265-72

41. Newville M 2001 IFEFFIT : interactive XAFS analysis and FEFF fitting. Journal of Synchrotron Radiation 8 322-4 
42. Ankudinov AL, Ravel B, Rehr JJ, Conradson SD 1998 Real-space multiple-scattering calculation and interpretation of $\mathrm{x}$-ray-absorption near-edge structure. Phys Rev $B \mathbf{5 8 ~ 7 5 6 5 - 7 6}$

43. Ravel B, Newville M 2005 ATHENA, ARTEMIS, HEPHAESTUS: data analysis for X-ray absorption spectroscopy using IFEFFIT. Journal of Synchrotron Radiation 12 537-41

44. Solera JA, García J, Proietti MG 1995 Multielectron excitations at the L edges in rare-earth ionic aqueous solutions. Phys Rev B 51 2678-86

45. Fonda E, Andreatta D, Colavita PE, Vlaic G 1999 EXAFS analysis of the L3 edge of Ce in CeO2: effects of multi-electron excitations and final-state mixed valence. Journal of Synchrotron Radiation 6 34-42

46. Romeo M, Bak K, El Fallah J, Le Normand F, Hilaire L 1993 XPS Study of the reduction of cerium dioxide. Surface and Interface Analysis 20 508-12

47. Bianconi A, Marcelli A, Dexpert H, Karnatak R, Kotani A, Jo T, et al. 1987 Specific intermediatevalence state of insulating $4 f$ compounds detected by $L_{3} x$-ray absorption. Phys $\operatorname{Rev} B 35$ 806-12

48. Soldatov AV, Ivanchenko TS, Della Longa S, Kotani A, Iwamoto Y, Bianconi A 1994 Crystal-structure

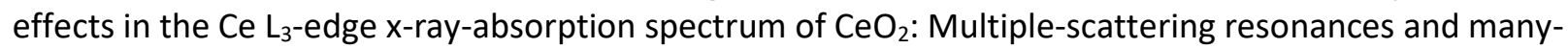
body final states. Phys Rev $B \mathbf{5 0}$ 5074-80

49. Finkelstein LD, Postnikov AV, Efremova NN, Kurmaev EZ 1992 X-ray Ce LIII absorption in CeO2 and BaCeO3: experiment and interpretation on the basis of LMTO band structure calculations. Materials Letters $14115-8$

50. Perebeinos V, Chan S-W, Zhang F 2002 'Madelung model' prediction for dependence of lattice parameter on nanocrystal size. Solid State Communications 123 295-7

51. Luches P, Pagliuca F, Valeri S, Boscherini F 2013 Structure of Ultrathin $\mathrm{CeO}_{2}$ Films on Pt(111) by Polarization-Dependent X-ray Absorption Fine Structure. J Phys Chem C 117 1030-6

52. Ahmed AS, Azam A, Muhamed Shafeeq M, Chaman M, Tabassum S 2012 Temperature dependent structural and optical properties of tin oxide nanoparticles. Journal of Physics and Chemistry of Solids $\mathbf{7 3}$ 943-7

53. Sheth J, Chen D, Kim JJ, Bowman WJ, Crozier PA, Tuller HL, et al. 2016 Coupling of strain, stress, and oxygen non-stoichiometry in thin film $\mathrm{Pr}_{0.1} \mathrm{Ce}_{0.9} \mathrm{O}_{2-\delta}$. Nanoscale $816499-510$ 